\title{
Optimal control of strong-field ionization with time-dependent density-functional theory
}

\author{
Maria Hellgren, ${ }^{1,2}$ Esa Räsänen, ${ }^{3}$ and E. K. U. Gross ${ }^{1}$ \\ ${ }^{1}$ Max-Planck Institute of Microstructure Physics, Weinberg 2, 06120 Halle, Germany \\ ${ }^{2}$ SISSA, International School for Advanced Studies, via Bonomea 265, 34136 Trieste, Italy \\ ${ }^{3}$ Department of Physics, Tampere University of Technology, FI-33101 Tampere, Finland
}

(Dated: July 22, 2018)

\begin{abstract}
We show that quantum optimal control theory (OCT) and time-dependent density-functional theory (TDDFT) can be combined to provide realistic femtosecond laser pulses for an enhanced ionization yield in many-electron systems. Using the $\mathrm{H}_{2}$-molecule as a test case, the optimized laser pulse from the numerically exact scheme is compared to pulses obtained from OCT+TDDFT within the TD exact-exchange (TDEXX) and the TD local-density approximation (TDLDA). We find that the TDDFT-pulses produces an ionization yield of up to $50 \%$ when applied to the exact system. In comparison, pulses with a single frequency but the same fluence typically reach to yields around $5-15 \%$, unless the frequency is carefully tuned into a Fano-type resonance that leads to $\sim 30 \%$ yield. On the other hand, optimization within the exact system alone leads to yields higher than $80 \%$, demonstrating that correlation effects beyond the TDEXX and TDLDA can give rise to even more efficient ionization mechanisms.
\end{abstract}

PACS numbers: 31.15.ee, 31.15.vj, 32.80.Qk, 33.80.Rv

\section{INTRODUCTION}

Developments in ultrafast science on the electronic timescale have been impressive during the past few years [1]. In particular, manipulation of both intense infrared and weak extreme ultraviolet pulses has led to innovative schemes to measure time delays in the attosecond range [2, 3]. It is expected that the ongoing advances will soon open a path into monitoring and controlling real-time electron dynamics.

In addition to measurements on the electronic timescale, subcycle pulse shaping has recently become possible. Wirth and co-workers [4] have generated synthesized laser pulses by combining subcycle transients across the infrared, visible, and ultraviolet regimes. In each regime, respectively, the chirp, carrier envelope phase, time delay, and energy (beam size) can be controlled before the final pulse is reconstructed.

The advances mentioned above bring the applications of optimized control schemes - such as quantum optimal control theory [5-7] (OCT) - to a new level of practical relevance. In atomic physics, OCT has been previously applied to design laser pulses for, e.g., enhanced [8] and suppressed ionization [9]. For molecular processes the range of applications is significantly larger covering, e.g., dissociation [10], chemical design [11], and molecular switches [12]. These research lines within OCT among other applications are described in a recent review by Brif et al. 7]

On the theoretical side, a single-active-electron approximation is usually employed [13] with the full treatment of many-electron effects being limited to numerical investigations on very small systems. With the aim of studying larger and more complex systems, timedependent density-functional theory [14, 15] (TDDFT) has emerged as a computationally efficient method that can, in principle, exactly deal with the dynamics of the full many-electron system. Within TDDFT the exact time-dependent density is obtained from a fictitious system of noninteracting electrons moving in an effective time-dependent Kohn-Sham (KS) potential. The KS potential is a unique functional of the density which, in practice, must be approximated. Over the past years different approximation schemes have been developed and tested showing both promise and future challenges [16 20]. In addition, very recently, the inverse problem, i.e., finding the field that drives the many-particle system to a desired outcome, has been formally solved within a combination of OCT and TDDFT [21].

In the present work we take steps in the practical validation of this combination by testing how fields optimized in the TDDFT framework (with different functionals) perform when applied in the numerically exact time-dependent Schrödinger equation. This question is of particular relevance from the experimental point of view; namely, when considering a many-electron system beyond the capabilities of a numerically exact treatment, can we design usable laser pulses with TDDFT+OCT to be used in an experiment for an enhanced effect? Our response will be affirmative, but with important reservations as will be discussed.

We focus on maximizing the total ionization of a model $\mathrm{H}_{2}$-molecule. First, a target functional in terms of the density is formulated and carefully validated. As our main result we show that the application of OCT in conjunction with TDDFT produces pulses that - when used in the exact system - lead to considerably higher ionization yields than non-optimized single-frequency fields with the same fluence. However, the lack of correlation effects beyond the simple approximations in the used adiabatic functionals is shown to affect the yield in a negative way. This effect is further exemplified by calculating 
the ionization yield of $\mathrm{H}_{2}$ as a function of the (single) photon frequency.

The paper is organized as follows. In Sec. II we introduce the model $\mathrm{H}_{2}$-system as well as the OCT scheme. In Sec. III we present our main results for the optimization of the ionization yield with different $\mathrm{XC}$ functionals in TDDFT and compare with the numerically exact scheme. We also investigate the single-frequency pulses which further underline the importance of correlation effects. The paper is summarized in Sec. IIIC.

\section{SYSTEM AND METHODOLOGY}

\section{A. Model Hamiltonian}

The $\mathrm{H}_{2}$-molecule is modeled in terms of a onedimensional (1D) system with a soft-Coulomb interaction between the electrons [8, 22]. This model has been shown to capture many qualitative features of the true 3D molecule, which is enough also for our purposes. The time-independent Hamiltonian of this system is given in Hartree atomic units (a.u.) by

$$
H=\sum_{i=1}^{2}\left\{-\frac{1}{2} \frac{\partial^{2}}{\partial x_{i}^{2}}+V_{\mathrm{ext}}\left(x_{i}\right)\right\}+V_{e e}\left(x_{1}, x_{2}\right),
$$

where the nuclear potential is

$$
V_{\text {ext }}\left(x_{i}\right)=-\frac{1}{\sqrt{\left(x_{i}-R / 2\right)^{2}+a}}-\frac{1}{\sqrt{\left(x_{i}+R / 2\right)^{2}+a}},
$$

with $x_{i}$ being the position coordinate of electron $i$, and $a$ a "softening" parameter for the electron-nucleus interaction; in this work $a=0.9$. Due to the short duration of the laser pulse (a few fs) the nuclei are considered to be fixed at their equilibrium separation of $R=1.5$ a.u. The electron-electron interaction is given by a soft-Coulomb interaction according to

$$
V_{e e}\left(x_{i}, x_{j}\right)=\frac{1}{\sqrt{\left(x_{i}-x_{j}\right)^{2}+1}},
$$

so that here the softening parameter is one. The exact eigenstates and eigenenergies can be found by the exact diagonalization of $H$. Applying a time-dependent field implies solving the full time-dependent Schrödinger equation. In this work the system is assumed to be in the ground state $\Psi(t=0)=\Phi_{0}$ when the laser is switched on. It is easy to see that, numerically, solving a 1D twoelectron problem is equivalent to solving a one-electron problem in 2D. Such calculations can be carried out using the OCTOPUS code [23], which is our choice for all the results presented in this work.

\section{B. Kohn-Sham system}

The TDDFT description of the same system uses the existence of an independent-particle system evolving ac-
TABLE I: Ionization potentials from the HOMO eigenvalue and excitation energies in TDEXX and TDLDA as compared to the exact results (in eV). Also the 'bare' KS-EXX results are presented.

\begin{tabular}{c|c|c|c|c}
\hline \hline & Exact & TDEXX & KS-EXX & TDLDA \\
\hline $\mathrm{I}_{p}$ & 19.32 & 19.05 & 19.05 & 12.52 \\
$\Delta E_{1}$ & 12.25 & 12.52 & 10.34 & 11.70 \\
$\Delta E_{2}$ & 15.24 & 15.51 & 14.97 & - \\
\hline \hline
\end{tabular}

cording to the KS Hamiltonian,

$$
H^{\mathrm{KS}}=\sum_{i=1}^{2}\left\{-\frac{1}{2} \frac{\partial^{2}}{\partial x_{i}^{2}}+V_{\mathrm{KS}}[n]\left(x_{i}\right)\right\}
$$

that exactly reproduces the true interacting density

$$
n(x, t)=2 \int d x^{\prime}\left|\Psi\left(x, x^{\prime}, t\right)\right|^{2}
$$

Due to the Runge-Gross theorem [14] the density uniquely determines all observables as a function of time. The KS potential $V_{\mathrm{KS}}$ is normally split into the external $V_{\text {ext }}$, the Hartree $V_{\mathrm{H}}[n]$ and the exchange-correlation (XC) potential $V_{\mathrm{xc}}[n]$. If the external potential is timedependent, also $V_{\mathrm{H}}$ and $V_{\mathrm{xc}}$ become time-dependent. In this work we have tested two different approximations to $V_{\mathrm{xc}}$ : the TD exact-exchange (TDEXX) approximation and the TD local-density approximation (TDLDA). Both of these approximations are adiabatic, i.e., they depend only on the instantaneous density (not on its history). For two electrons the TDEXX approximation is equivalent to the time-dependent Hartree-Fock approximation of many-body perturbation theory and equals $V_{\mathrm{x}}(x, t)=-1 / 2 V_{\mathrm{H}}(x, t)=-1 / 2 \int d x^{\prime} V_{e e}\left(x, x^{\prime}\right) n\left(x^{\prime}, t\right)$. A $1 D$ version of the LDA for soft-Coulomb interactions has recently been developed by Helbig et al. [18].

The equilibrium properties calculated exactly as well as within TDEXX and TDLDA are summarized in Table I. The ionization energy $I_{p}$ is obtained from the highest occupied molecular orbital (HOMO) of the unperturbed KS system. We find that TDEXX is in good agreement with the exact result. Excitation energies can be calculated by means of linear response TDDFT 24] where only the first functional derivative $F_{\mathrm{xc}}=\mathrm{V}_{\mathrm{xc}} / \mathrm{n}$, the so-called XC kernel, enters. In TDEXX $F_{\mathrm{x}}=-1 / 2 V_{e e}\left(x, x^{\prime}\right)$ and we find that the two first excitation energies $\Delta E_{1}$ and $\Delta E_{1}$ agree well with the exact values. The "bare" KS excitation energies are also presented. TDLDA gives a rather poor ionization energy due to the exponential decay of the LDA-XC potential. The same property leads to only one empty bound state, which, on the other hand, is rather well described.

Even if TDEXX and TDLDA give a reasonable account of the ground -and low-lying excited states of the system, it remains to be tested to what extent non-linear 

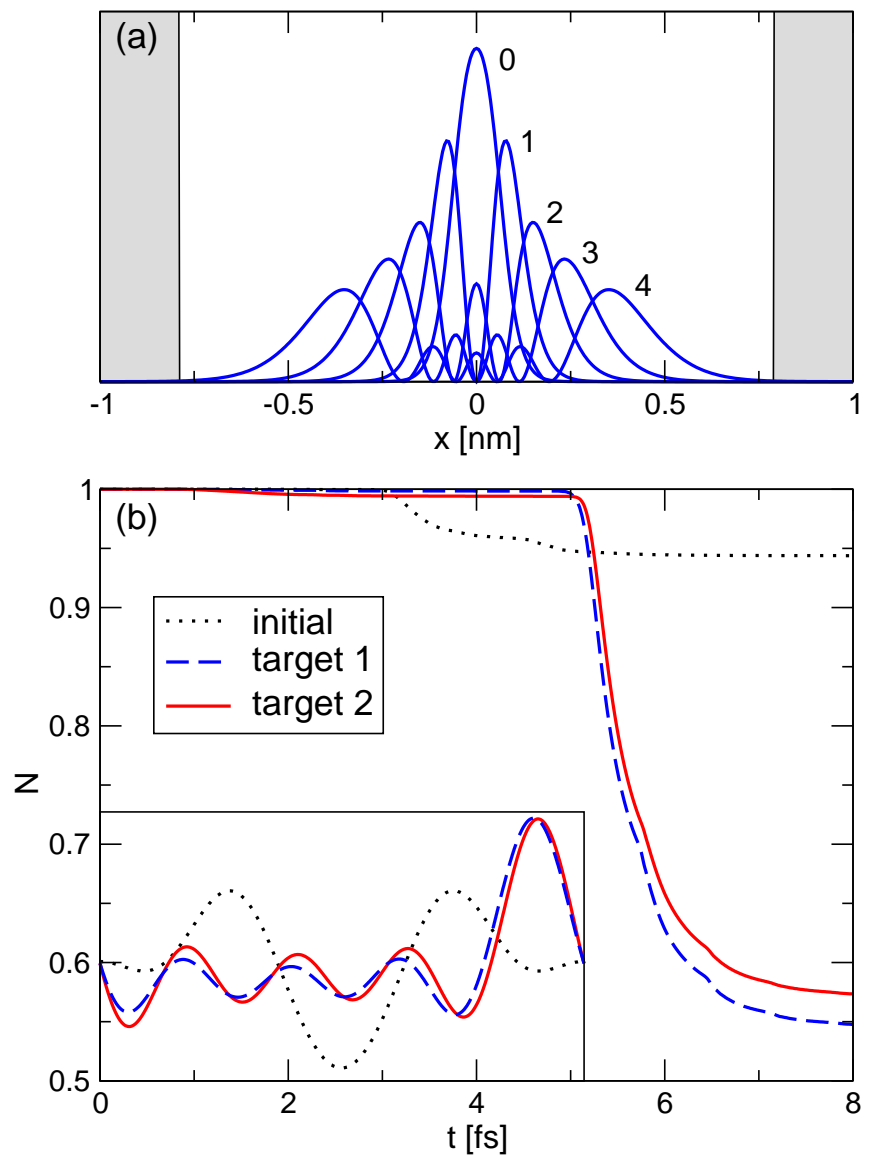

FIG. 1: (a) Probability densities of the five lowest eigenstates in a $1 \mathrm{D} \mathrm{H}_{2}^{+}$molecule. The first target for ionization is to exclude the occupation of these states ("target 1"), whereas the alternative target is to maximize the overlap between the density and the shaded region ("target 2"). (b) Electron number in $|x| \leq R$ as a function of time in the presence of the initial pulse (dotted line) and pulses optimized by using target 1 (dashed line) and target 2 (solid line). The pulse profiles are shown in the inset.

responses can be captured. Previous studies show that, e.g., the adiabatic approximation tend to detune resonances in the non-linear regime [18]. In the following we will investigate whether it is possible to find a common femtosecond laser pulse that can enhance the ionization yield in both the KS system and in the exact system.

\section{Pulse optimization and the target functional}

The laser pulse for the optimization is expressed as

$$
\varepsilon(t)=\sum_{n=1}^{M}\left[f_{n} \sqrt{\frac{2}{T}} \cos \left(\omega_{n} t\right)+g_{n} \sqrt{\frac{2}{T}} \sin \left(\omega_{n} t\right)\right],
$$

where the amplitudes $\left\{f_{n}, g_{n}\right\}$ are varied during the optimization. The number of allowed frequencies $M$ is determined by the cut-off frequency $\omega_{\max }$ and the pulse duration $T$, fixed to values $9.25 \mathrm{eV}$ and $5.3 \mathrm{fs}$, respectively.
This gives $M=12$. The cut-off frequency is thus chosen to be smaller than two times the ionization energy and smaller than the first excitation energy. As we will see this choice still allows for ionization via excited states. The amplitudes are constrained by keeping the fluence, i.e., the time-integrated intensity of the laser pulse fixed.

We apply OCT in a so-called direct-optimization scheme presented in detail in Ref. [8]. In practice, we maximize a merit function for a set of parameters of the laser pulse by using the derivative-free NEWUOA algorithm [25] between consecutive time-propagations. Expressing the pulse in a proper Fourier basis [8] guarantees that the conditions $\int_{0}^{T} d t \varepsilon(t)=0$ and $\epsilon(0)=\epsilon(T)=0$ are satisfied.

In order to maximize the ionization yield we need to formulate a target functional to be used in the OCT calculation. In Ref. [8] the ionization target was expressed as an exclusion of a set of lowest bound states. Here, in order to apply TDDFT, we need to write the target in terms of the density only. This gives us two choices: (i) we can minimize the density inside radius $R$ or (ii) we can maximize it outside $R$ at the end of the pulse. In case (i) we minimize the overlap between the density and a Heaviside step function of the form $-\Theta(R-|x|)$, whereas in case (ii) we maximize the overlap between the density and $\Theta(|x|-R)$. In principle, these cases are identical, but due to a finite simulation box and absorbing boundaries we resort to choice (i) - apart from the test case described below in this section. We set $R$ to be equal to the box radius (40 a.u.).

The ionization probability $P$ can be determined from the remaining density in the system in the long-time limit, i.e.,

$$
P=1-\frac{1}{2} \int_{-R}^{R} d x n(x, t \rightarrow \infty) .
$$

In practice we calculate $P$ at $T=8 \mathrm{fs}$, when the density has almost fully converged. As the pulse length is fixed to $5.3 \mathrm{fs}$, we thus continue the time-propagation after the field has been switched off, during which the density continues to evolve in the system.

In Fig. 1 we assess the validity of the density target described above by considering the ionization process of a 1D single-electron $\mathrm{H}_{2}^{+}$molecule. The peak intensity of the initial pulse is set to $10^{15} \mathrm{~W} / \mathrm{cm}^{2}$. Figure 1(a) shows the probability densities of the five lowest eigenstates. The first target operator ("target 1") is defined as $1-\sum_{i=0}^{4}\left|\Phi_{i}\right\rangle\left\langle\Phi_{i}\right|$, i.e., we are attempting to avoid the occupation of the five lowest states. This type of a target for ionization has been validated in previous studies [8]. Alternatively, "target 2" is defined from the density by maximizing the overlap with the shaded region in Fig. 1(a) corresponding to $\Theta(|x|-R)$ [case (ii) above]. To enable a direct comparison between the two targets in this example, we use here $R=15$ that approximately agrees with the spatial extent of the four lowest eigenstates [Fig. 1(a)]. As shown in Fig. 1(b), the results 
(a) Exact

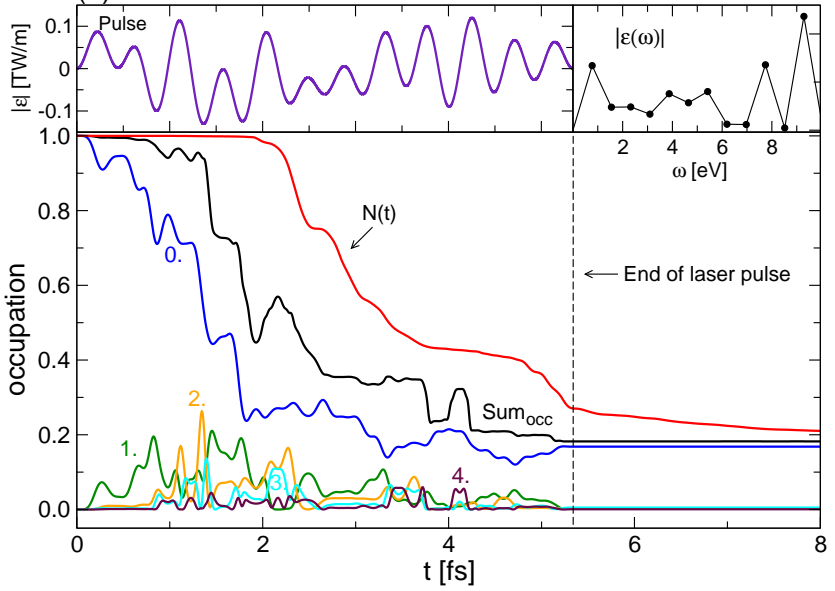

(c) TDLDA

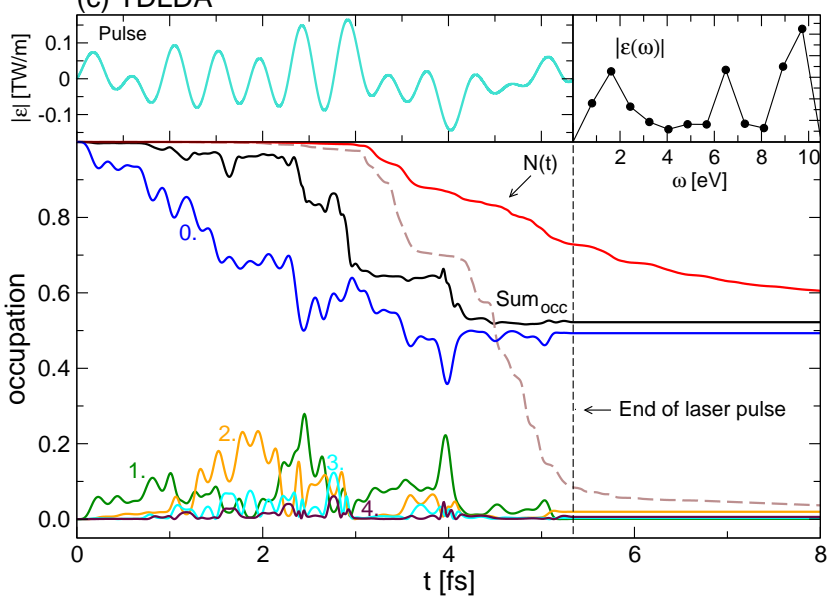

(b) TDEXX

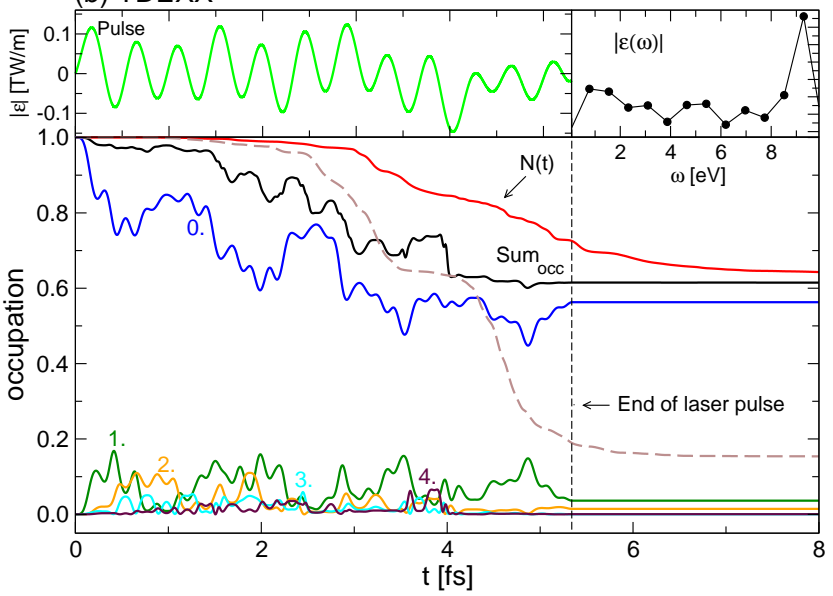

(d) KS-EXX

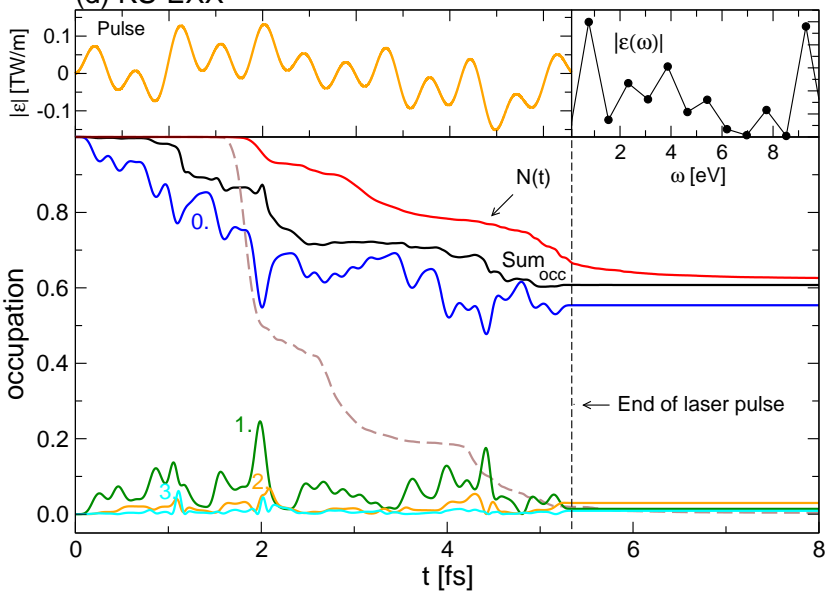

FIG. 2: Optimized laser pulses (upper left panel), their Fourier transforms (upper right panel), and the projections on the excited states as well as the number of particles as a function of time in the exact system (lower panel) from (a) exact optimization, (b) TDEXX, (c) TDLDA, and (d) KS-EXX. The dashed fade line in (b)-(d) is the number of particles $N(t)$ in the corresponding KS system under the influence of the same laser pulse.

for the decaying number of electrons $N$ in $|x| \leq R$ as a function of time, as well as for the optimized pulses (inset), are almost identical for these two targets. In both cases the ionization probability is significantly increased through optimization. We point out that here the high ionization yield results primarily from the high intensity peak near the end of the pulse. Detailed discussion on such an OCT process in the tunneling regime can be found in Refs. [8] and [9].

\section{RESULTS}

\section{A. Results for ionization}

Now we switch back to the original two-electron $\mathrm{H}_{2}$ system defined in Sec. IIA Figure 2(a) shows the results obtained from the optimization in the exact system. The optimal laser pulse and its Fourier trans- form are shown in the upper left and right panel, respectively. In the lower panel we show the evolution of the integrated density (normalized to one) in the system, $N(t)=(1 / 2) \int_{-R}^{R} d x n(x, t)$ (red solid line). Thus, in the long-time limit the ionization probability in Eq. (7) can be expressed as $P=1-N(t \rightarrow \infty)$. We also plot the projections $\left|\left\langle\Psi(t) \mid \Phi_{i}\right\rangle\right|^{2}[i=0, \ldots, 4]$, where $\Psi(t)$ is the time-evolved wave function and $\Phi_{i}$ denotes the $i$ :th eigenstate of the unperturbed system. The sum of the projections, corresponding to the total occupation of the four lowest eigenstates, is also shown. It can be expected that in the $t \rightarrow \infty$ limit $N(t)$ approaches the sum of the occupations. This is due to the fact that after the pulse has been switched off, the part of the electron density that is not bound in the lowest states eventually propagates into the absorbing boundaries. This expectation is confirmed by all the results below.

We see in Fig. 2(s) that already at $t=2$ fs the probability of an electron being ionized is around $50 \%$. The 
ionization process involves mainly $\Phi_{1}$ and $\Phi_{2}$, starting with an increased occupation of $\Phi_{1}$ as the ground-state is being deoccupied. Around $t=1$ fs we find a transfer from $\Phi_{1}$ to $\Phi_{2}$, just before the laser reaches its peak intensity where ionization is pronounced. A similar effect is repeated thereafter. During the remaining pulse duration, a smooth depopulation of the bound states takes place, and at the end only $20 \%$ of the ground state is occupied. Around $t=8 \mathrm{fs}$, the $N(t)$ curve converges to the sum of the projections and we find the yield to be $80 \%$. This should be compared with the random initial pulse giving a yield of less than $20 \%$.

In Fig. 2(b) we show the results obtained by optimizing the laser pulse in the KS system within the TDEXX approximation. By applying this laser pulse to the exact system we find the results of the lower panel. The dashed fade line in the background is the $N(t)$ curve of the KS density under the influence of the same laser pulse, leading to a final yield of $80 \%$ in the KS system. When applied to the exact system the same laser pulse gives a yield of around $40 \%$. This shows that the densities in the exact and in the TDEXX systems behave quite differently, and that the pulse optimized in the TDEXX scheme has only a limited ionization effect on the exact system. Despite its limitations, the KS optimization is seen to produce a better pulse than the random initial guess and as we shall see later than pulses containing only a single frequency. The projections on the excited states of the exact system show that also with the TDDFT pulse the excited states are involved. The major difference as compared to the exact case is that the ground state gets repopulated after being depopulated. The transfer to the second excited state or to the continuum is therefore not complete as in the exact case. This oscillating behavior is seen throughout the pulse duration and it appears to prevent complete ionization.

Figure 2(c) shows the results from the TDLDA optimization. In this case we are able find a slightly higher yield of $50 \%$ when the pulse is applied to the exact system. We also see that the oscillating behavior is reduced and the second excited state is better exploited than in the TDEXX case. We have also performed an optimization keeping the KS-EXX potential fixed in time [Fig. $2(d)$. In that case only the first excited state is used. The final yield is, however, still as high as $40 \%$.

We point out that the obtained pulses are not unique, which is a common feature of all OCT studies. We can therefore not entirely exclude the possibility that there is a pulse that can produce the same yield in the approximate as in the exact system. The algorithm finds slightly different pulses depending on the initial condition for the optimization.

\section{B. Single-frequency pulses}

In order to gain further insight into the ionization process of $\mathrm{H}_{2}$ we test the effect of laser pulses containing

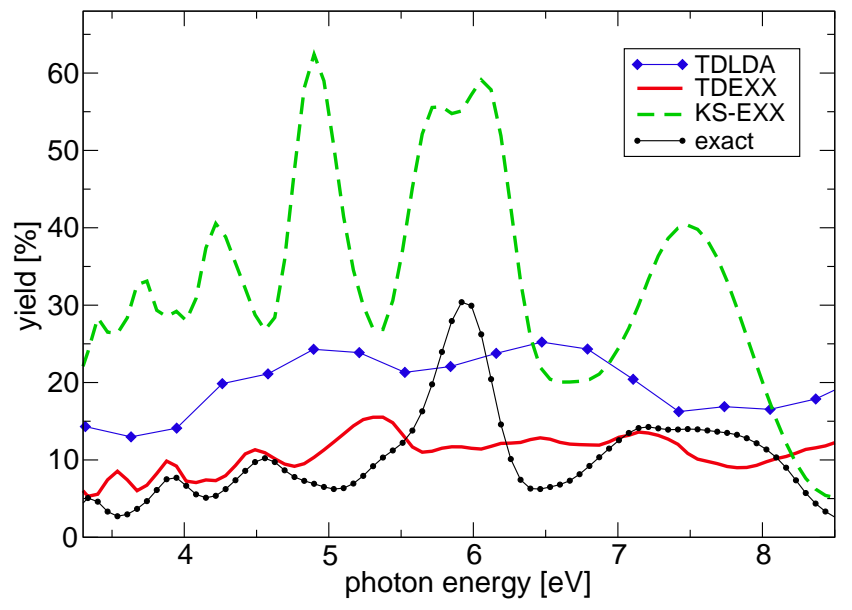

FIG. 3: Numerically exact result of the total ionization yield in a one-dimensional $\mathrm{H}_{2}$ molecule with a soft-Coulomb interaction as a function of laser frequency (photon energy). The pulse duration is $2.65 \mathrm{fs}$ and the peak intensity is $5 \times 10^{14}$ $\mathrm{W} / \mathrm{cm}^{2}$.

only a single frequency according to $\varepsilon(t)=f(t) \cos (\omega t)$, where the pulse envelope is given by $f(t)=F_{0} \cos [\pi / 2(t-$ $3 T) / T$. The amplitude $F_{0}$ is chosen to produce a peak intensity of $5 \times 10^{14} \mathrm{~W} / \mathrm{cm}^{2}$, and the pulse length is $T=2.65 \mathrm{fs}$. The total propagation time is $8 \mathrm{fs}$, so that $f(t)=0$ at $t>T$. The fluence is equal to OCT processes described above. The whole frequency range below the cut-off frequency chosen for the optimized laser pulses is scanned, and the ionization yield is determined by integrating the density in the simulation box as explained in Sec. IIC.

The yield as a function of frequency is plotted in Fig. 3 . The exact result (black-dotted curve) is compared to the TDEXX result (red solid line), the TDLDA (blue dashed line), and the KS-EXX (green dashed line). Notice that if the exact time-dependent KS potential were used, the KS yield would be equal to the exact yield since only the density is needed to determine the ionization yield. Distinct peaks are found at certain frequencies. They are strongly emphasized in the noninteracting KS-EXX spectrum, which can be considered as our zeroth-order approximation with respect to the Coulomb interaction. This choice of a zeroth-order system leads to the correct description of the ionization energy of the first electron, but misses the fact that the second electron should be more bound and much harder to ionize. The role of the time-dependent effective potential is to simulate this effect and reduce the yield, here by roughly a factor of four. This effect is rather well reproduced by the TDEXX. The peaks are also somewhat shifted in the TDEXX and hence in better agreement with the exact result, at least at low frequencies.

At around $\omega=6 \mathrm{eV}$, we find a sharp peak in the exact spectrum that exhibits an asymmetric Fano-type peak. Its location is very close to a half of the first discrete excitation energy at $\omega=12.25 \mathrm{eV}$. We note, however, that 
the other peaks cannot be associated to the excitation energies of equilibrium system due to the large amplitude of the field.

In the TDEXX results we find similarities to the exact result, especially in the low-energy regime, but no clear signatures of a Fano-type resonance. This suggests that correlation effects are important to describe this resonance. The TDLDA curve shows a suppression of the yield but the effect is less accurate as compared to TDEXX. Also here the resonance is missing.

The maximum yield we can obtain using a laser pulse with a single frequency and peak intensity of 500 $\mathrm{TW} / \mathrm{cm}^{2}$ is around $30 \%$. Thus, even if we were able to locate the resonance, the yield is still lower than obtained in the OCT procedure (within all the tested approximations in TDDFT) that allows for more frequencies. This motivates the use of OCT for an enhanced yield as opposed to an optimal single-frequency pulse.

\section{Summary}

We have applied quantum optimal control theory in conjunction with time-dependent density-functional theory (TDDFT) to examine enhanced ionization of a model $\mathrm{H}_{2}$ molecule. First we have validated the use of a density- based target for the maximum ionization in the TDDFT framework. According to our main results, pulse optimization within the (adiabatic) exact-exchange formalism and the local-density approximation provide reasonable pulses for enhanced ionization: when those pulses are applied to the exact system the yield is considerably increased with respect to the initial random guess or with respect to the single-frequency result. However, we have found that these functionals are unable to capture complicated correlation effects in the system. The presence of these effects becomes clear in the analysis of the exact ionization yield as a function of the pulse frequency, revealing, e.g., a sharp resonance. In conclusion, TDDFT may be used as the first attempt to optimize strong-field effects in atomic systems, but further work is needed to construct more accurate functionals to account for manyparticle phenomena at a deeper level.

\section{Acknowledgments}

This work was supported by the Academy of Finland and the European Community's FP7 through the CRONOS project, grant agreement no. 280879. We are grateful to CSC - the Finnish IT Center for Science - for computational resources.
[1] F. Krausz and M. Ivanov, Rev. Mod. Phys. 81, 163 (2009).

[2] M. Schultze, M. Fieß, N. Karpowicz, J. Gagnon, M. Korbman, M. Hofstetter, S. Neppl, A. L. Cavalieri, Y. Komninos, Th. Mercouris, C. A. Nicolaides, R. Pazourek, S. Nagele, J. Feist, J. Burgdörfer, A. M. Azzeer, R. Ernstorfer, R. Kienberger, U. Kleineberg, E. Goulielmakis, F. Krausz, and V. S. Yakovlev, Science 328, 1658 (2010).

[3] K. Klünder, J. M. Dahlström, M. Gisselbrecht, T. Fordell, M. Swoboda, D. Guénot, P. Johnsson, J. Caillat, J. Mauritsson, A. Maquet, R. Taïeb, and A. LHuillier, Phys. Rev. Lett. 106, 143002 (2011).

[4] A. Wirth, M. Th. Hassan, I. Grguras, J. Gagnon, A. Moulet, T. T. Luu, S. Pabst, R. Santra, Z. A. Alahmed, A. M. Azzeer, V. S. Yakovlev, V. Pervak, F. Krausz, and E. Goulielmakis, Science 334, 195 (2011).

[5] A. P. Peirce, M. A. Dahleh, and H. Rabitz, Phys. Rev. A 37, 4950 (1988); R. Kosloff, S. A. Rice, P. Gaspard, S. Tersigni, and D. J. Tannor, Chem. Phys. 139, 201 (1989).

[6] J. Werschnik and E. K. U. Gross, J. Phys. B: Atom. Mol. Opt. Phys. 40, R175 (2007).

[7] C. Brif, R. Chakrabarti, and H. Rabitz, New J. Phys. 12, 075008 (2010).

[8] A. Castro, E. Räsänen, A. Rubio, and E. K. U. Gross, Europhys. Lett. 87, 53001 (2009).

[9] E. Räsänen and L. B. Madsen Phys. Rev. A 86, 033426 (2012).

[10] K. Krieger, A. Castro, and E. K. U. Gross, Chem. Phys. 391, 50 (2011).

[11] T. Laarmann, I. Shchatsinin, P. Singh, N. Zhavoronkov,
M. Gerhards, C. P. Schulz, and I. V. Hertel, J. Chem. Phys. 127, 201101 (2007).

[12] D. Geppert, L. Seyfarth, and R. de Vivie-Riedle, Appl. Phys. B 79, 987 (2004).

[13] M. Awasthi, Y. V. Vanne, A. Saenz, A. Castro, and P. Decleva, Phys. Rev. A 77, 063403 (2008).

[14] E. Runge and E. K. U. Gross, Phys. Rev. Lett. 52, 997 (1984).

[15] For a review, see C. Ullrich, Time-Dependent DensityFunctional Theory: Concepts and Applications (Oxford University Press, 2012).

[16] M. Mundt and S. Kümmel Phys. Rev. A 74, 022511 (2006).

[17] M. Thiele, E. K. U. Gross, and S. Kümmel Phys. Rev. Lett. 100, 153004 (2008).

[18] N. Helbig, J. I. Fuks, M. Casula, M. J. Verstraete, M. A. L. Marques, I. V. Tokatly, and A. Rubio, Phys. Rev. A 83, 032503 (2011).

[19] J. I. Fuks, N. Helbig, I. V. Tokatly, and A. Rubio Phys. Rev. B 84, 075107 (2011).

[20] D. Hofmann, T. Körzdörfer, and S. Kümmel Phys. Rev. Lett. 108, 146401 (2012).

[21] A. Castro, J. Werschnik, and E. K. U. Gross, Phys. Rev. Lett. 109, 153603 (2012).

[22] J. Javanainen, J. Eberly, and Q. Su, Phys. Rev. A 38, 3430 (1988).

[23] M. A. L. Marques, A. Castro, G. F. Bertsch and A. Rubio, Comp. Phys. Comm. 151, 60 (2003); A. Castro, H. Appel, M. Oliveira, C. A. Rozzi, X. Andrade, F. Lorenzen, M. A. L. Marques, E. K. U. Gross, and A. Rubio, Phys. Stat. Sol. (b) 243, 2465 (2006). 
[24] M. Petersilka, U. J. Gossmann, and E. K. U. Gross, Phys. Rev. Lett. 76, 1212 (1996).

[25] M. J. D. Powell, Large-Scale Nonlinear Optimization

(Springer, New York, 2004), pp. 255297. 